\title{
SIMULATION OPTIMISATION FOR OPERATING ROOM SCHEDULING
}

\author{
Baesler, F.; Gatica, J. \& Correa, R. \\ Universidad del Desarrollo, College of Engineering, Av. Plaza 700, Santiago, Chile \\ E-Mail: fbaesler@udd.cl,gaticafuentes@gmail.com,RCorr004@codelco.cl
}

\begin{abstract}
This paper presents a case study on operating room scheduling in a small hospital in Chile. Patient flow was represented using a discrete-event simulation model that considered the randomness associated with the primary activities of the entire process, which includes pre- and posthospitalisation, surgery, surgery setup and recovery. A simulated annealing algorithm was implemented and connected to the simulation model to search for better patient schedules. Additionally, three dispatching rules, Shortest Processing Time (SPT), Longest Processing Time (LPT) and First-In, First-Out (FIFO) were used. The results showed that the simulated annealing approach, based on the Cmax objective function, obtained schedules that were $18 \%$ better than the hospital's scheduling practices. The utilisation of dispatching rules also has a significant effect in the Cmax indicator. The SPT rule performed better than the hospital schedule in two of the three experiments considered in the study.

(Received in March 2014, accepted in January 2015. This paper was with the authors 1 month for 1 revision.)
\end{abstract}

Key Words: Simulation Optimisation, Simulated Annealing, Operating Room, Scheduling

\section{INTRODUCTION}

Operating room scheduling is one of the many complexities encountered in hospitals. This activity is typically performed semi-manually based on experience and is often extremely time consuming. Operating room scheduling should be coordinated with the allocation of resources associated with the operating rooms, such as pre- and post-surgical beds and human resources, such as nurses and medical hours. Performing this activity well is crucial in reducing patient's waiting lists, improving the service levels provided to patients, reducing health equity gaps and improving the perception of the target population. Generally, these problems are complex and difficult to solve manually due to their combinatorial nature, which could have millions of possible solutions (or different schedules), making it impossible to obtain optimal solutions manually. The literature reports attempts to address this problem from different perspectives. Most of the literature models the problem as a deterministic environment. In those cases, the use of mathematical programming approaches is extremely common. Good reviews can be found in [1] and [2].

In the particular field of simulation-based scheduling, it is possible to find studies associated with industry applications. Several examples can be found in [3], which evaluated a total of 44 dispatching rules in an automotive job shop simulation case study. The authors used single and hybrid rules, and the best result was obtained with the Most Total Work Remaining rule. In [4], the authors developed evolutionary simulation-based heuristics to construct near-optimal solutions for dispatching rule allocation for a case study analysis of a discrete-event simulation model of a commercial offset printing production system. [5] presents an optimisation via simulation approach to solve dynamic, flexible job shop scheduling problems using genetic algorithms. The results revealed that optimisation using the simulation approach is a good way to solve dynamic, flexible job shop scheduling problems, which generally takes the NP-Hard form.

Simulation-based scheduling in the healthcare field has been analysed by several authors. In [6], the authors present a combined optimisation simulation approach to the master surgical 
scheduling problem using a mix integer programming model to find optimal solutions and used a discrete-event simulation to test its robustness. A similar situation, where a mix integer programming model and a Monte Carlo simulation are combined, is presented in [7]. In [8], the authors combine a simulation model and a genetic algorithm to search for better schedules for hospital admissions. [9] proposed an approach to optimise surgery start times for a single operating room with stochastic operation durations. The authors used response surface methodology to optimise the simulated schedules. [10] presents an approach for appointment scheduling using a discrete simulation and a random search algorithm to search for solutions. The results showed that in small problems, it is possible to obtain good schedules in a short amount of time. Other work related to appointment scheduling is presented in [11]. In this study, the authors did not use a search algorithm but instead, evaluated nine different schedules via a simulation of an Ear, Nose and Throat (ENT) outpatient department. The results show that the selection of a good schedule can reduce waiting times by a significant amount. In [12], a simulation model of an operating room was created that uses a bin packing algorithm to schedule the patients in time blocks. The objective of that study was to maximise the operating room use. A discrete-event simulation model of an outpatient clinic of a medical centre in Indianapolis is presented in [13], where the authors analysed the performance of different open-access scheduling configurations under various clinical environments in terms of patient waiting time, patient rejection rate and clinic use. [14] represented the operating room as a parallel machine problem using a discrete-event simulation model. Three dispatching rules were evaluated, where the best performance was obtained with the Longest Expected Processing with Setup Time (LEPST) rule. A similar work using simulation and dispatching rules is presented by [15], where the authors analysed the process by considering the number of pre- and post-recovery beds, not just the availability of the operating rooms. In this case, the best rule was SPT.

Most of the approaches presented in literature that used simulations focused on comparing different dispatching rules. Only a few cases applied a search algorithm to find near optimal schedules. In this work, we present a case study where simulated annealing is used as the search mechanism to find good schedules by considering all the primary resources related to a surgery. The number of pre- and post-surgery beds, number of operating rooms and healthcare human resources are included in the simulation model to represent the entire cycle as realistically as possible and include the randomness inherent in all the activities.

The paper is structured as follows: an introduction section, a methodology section, case study problem description, data analysis, optimisation process, results and conclusions.

\section{METHODOLOGY}

A scheduling problem is a complex combinatorial problem, where each possible patient combination represents a different solution. If $n$ is the number of patients that exist, there can be $n$ ! different schedules. The time required to evaluate all of the $n$ ! solutions is prohibitive in most cases and even more complex for the problem presented in this paper because each possible solution is represented by a simulation scenario. For this reason, optimisation techniques are used to find optimal or near optimal solutions without solving all the possible combinations. One strategy is to connect the simulation model and a heuristic for searching for better solutions; this is depicted in Fig. 1.

Fig. 1 shows the simulation optimisation scheme. One initial set of patients is scheduled in a particular way, generally randomly, and is simulated to estimate a performance measure of this particular schedule. These results are used by a search method to propose a new schedule that is simulated by the model. This iterative cycle is repeated until a termination condition is met. 


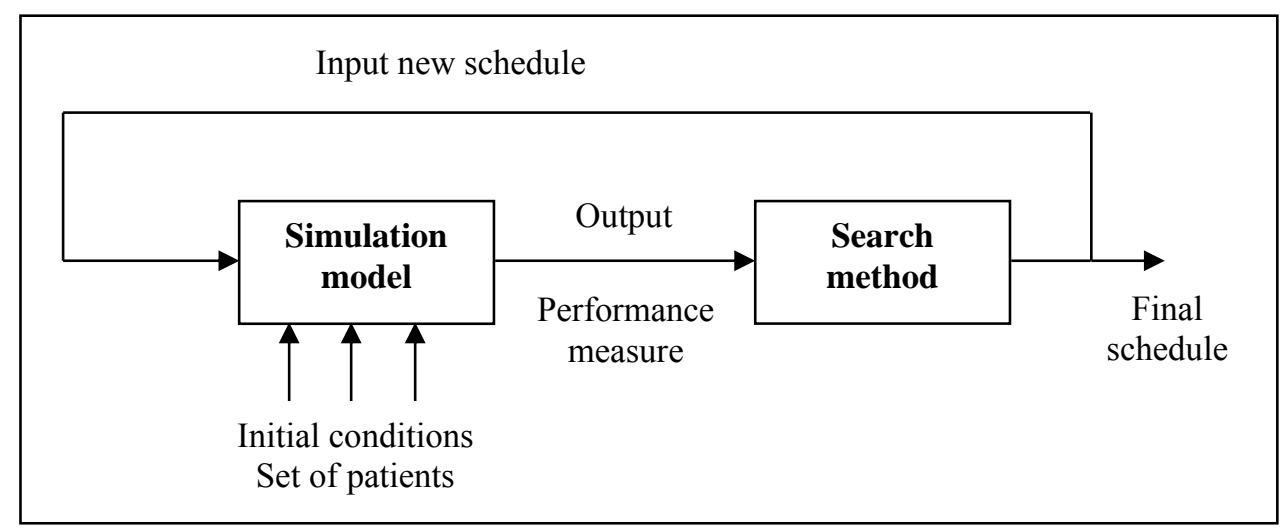

Figure 1: Simulation optimisation iterative scheme.

This study uses simulated annealing as the search algorithm. This method was introduced by [16], popularised by [17] and has been used in several problems. Several examples of simulated annealing used in scheduling environments can be found in [18] for job shop scheduling, [19] for project scheduling, [20] for vehicle design scheduling, [21] for parallel machine scheduling and [22] for flow shop scheduling. In simulation optimisation, simulated annealing has been used as search engine in some cases. A multiobjective optimisation approach based on simulated annealing and discrete event simulation was presented by [23] for the maintenance scheduling problem of a fleet of fighter aircraft. Simulation optimisation of police patrol districting plans is studied by [24]. They compare simulated annealing and response surface methodology concluding that good districting plans can be generated more efficiently with designed experiments and provide better alternatives for police departments. [25] developed a deterministic simulation model connected to a simulated annealing search engine to design of best possible routes for a set of trucks serving multiple loggers and mills. Good results are obtained comparing the methodology against real data provided by a log delivery trucking firm.

\subsection{Solution codification}

To implement the simulated annealing algorithm, a coding scheme is required that represents each solution. In this case, a solution represents a particular patient schedule that can be represented as a list of patients who must be served in the order of the list. An example of 6 patients is presented in Fig. 2, where patient 5 is served first, then patient 1 and so on.

\begin{tabular}{|l|l|l|l|l|l|}
\hline P5 & P1 & P4 & P3 & P6 & P2 \\
\hline
\end{tabular}

Figure 2: Solution codification.

The objective function used in this project was the minimisation of the maximum completion time (Cmax), which means that each schedule run in the simulation model will generate a C $\max$ that represents the total time required to serve the entire batch of patients. Because simulated annealing is a local search technique, the concept of a neighbour must be defined. In this problem, a neighbour is a solution that is "close" to an original solution. The neighbourhood exploration definition used in this research was pairwise exchange, which means that two patients of the same schedule are selected randomly to exchange places, which generates a new neighbour solution. Fig. 3 illustrates an original solution and the corresponding neighbour for a problem consisting of 6 patients. Patients 2 and 6 were randomly selected for exchange. 


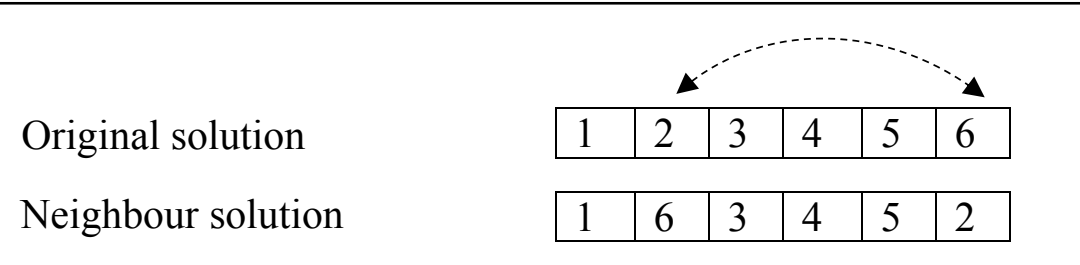

Figure 3: Neighbourhood representation.

\section{CASE STUDY}

The hospital under study is a small hospital located in Chile. The hospital contains 38 hospital beds, 6 recovery beds and 3 operating rooms. One of these rooms is reserved for emergency patients. Fig. 4 presents the flow for a scheduled surgical patient.

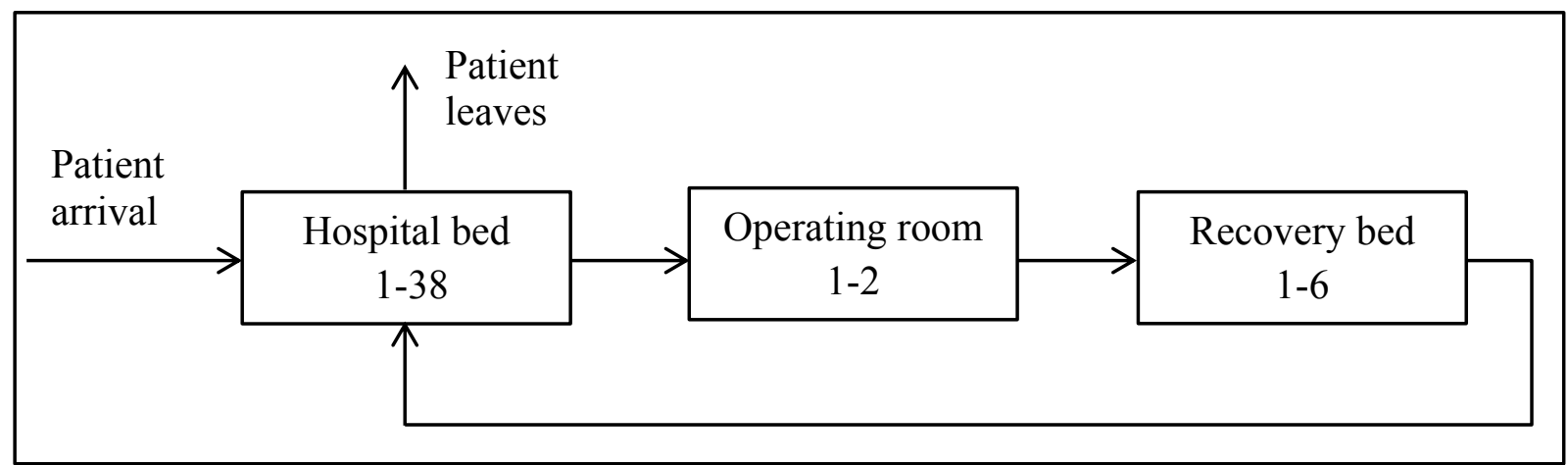

Figure 4: Surgical flow.

Fig. 4 shows that every scheduled patient has to be assigned to one of the 38 hospital beds; afterwards, the surgery is performed in one of the two operating rooms. After surgery, the patient uses one of the 6 recovery beds. Finally, the patient returns to the hospital bed for a certain number of days depending on the type of surgery.

\subsection{Patient categories}

The hospital performs 85 different types of surgeries; however, 25 of the surgery types represent $90 \%$ of the total surgeries performed each year. For that reason, our analysis was concentrated in those 25 surgery categories. The patient flow was simplified to four primary activities: surgery, surgery setup, recovery and hospitalisation. For each of the 25 surgery categories, a statistical analysis was performed based on a data base of 2500 patients. The duration of each one of these activities was analysed and fitted using the KolmogorovSmirnov test, where $P$ values greater than 0.05 were obtained in all of the cases, except in 2 categories where empirical distributions were used. For the parameter, number of hospitalisation days, a discrete empirical distribution was used. The resulting distributions for the surgery, setup and recovery times are presented in Table I.

It is possible to appreciate that for a same activity, different statistical distributions are obtained in each patient category. This issue is common in healthcare because the duration of one activity could differ significantly among types of surgeries. For example, the surgery time distribution could present different types of tails because the risk of an unexpected event is not the same for each type of surgery. The recovery time could also be different because the type of anaesthesia used in the surgery or other issues such as patient condition or age may prolong the duration of this activity. 
Table I: Activity distributions.

\begin{tabular}{|c|c|c|c|}
\hline $\begin{array}{l}\text { Surgery } \\
\text { category }\end{array}$ & Setup time [min] & Surgery time [min] & Recovery time [min] \\
\hline 1 & $5+56 * \operatorname{BETA}(0.25,0.724)$ & $\operatorname{TRIA}(25,57.7,71)$ & $55+\mathrm{EXPO}(17.8)$ \\
\hline 2 & $\operatorname{EXPO}(15.8)$ & $\operatorname{TRIA}(30,60,150)$ & $\operatorname{NORM}(67.5,24.6)$ \\
\hline 3 & $\operatorname{WEIB}(32.9,0.55)$ & $10+61 * \operatorname{BETA}(0.98,0.641)$ & $\operatorname{NORM}(65.6,26.3)$ \\
\hline 4 & $\operatorname{EXPO}(13.7)$ & 45+WEIB $(47.8,1.29)$ & $\operatorname{TRIA}(10,84,115)$ \\
\hline 5 & $60 * \operatorname{BETA}(0.76,1.89)$ & $5+\operatorname{WEIB}(26.4,1.22)$ & $5+\operatorname{EXPO}(21)$ \\
\hline 6 & $\operatorname{EXPO}(24.5)$ & $35+\operatorname{EXPO}(67.2)$ & $30+155^{*} \operatorname{BETA}(0.633,0.917)$ \\
\hline 7 & 5+EXPO(11.9) & $\operatorname{UNIF}(24.5,70.5)$ & $\operatorname{TRIA}(5,82.7,105)$ \\
\hline 8 & $90 * \operatorname{BETA}(1.25,1.35)$ & $\operatorname{NORM}(93.3,26.4)$ & $\operatorname{NORM}(105,32.3)$ \\
\hline 9 & EMPIRICAL & EMPIRICAL & EMPIRICAL \\
\hline 10 & EMPIRICAL & EMPIRICAL & 4.5+WEIB $(5.89,0.717)$ \\
\hline 11 & $225^{*} \operatorname{BETA}(1.11,4.17)$ & 5+WEIB $(59.6,1.72)$ & $\operatorname{NORM}(101,42.1)$ \\
\hline 12 & $\operatorname{UNIF}(10,145)$ & $40+96 * \operatorname{BETA}(0.693,1.16)$ & $15+140 * \operatorname{BETA}(0.494,0.481)$ \\
\hline 13 & $165 * \operatorname{BETA}(0.929,2.25)$ & $10+306 * \operatorname{BETA}(1.66,5.12)$ & $\operatorname{NORM}(107,47.4)$ \\
\hline 14 & $\operatorname{UNIF}(10,36)$ & $25+\mathrm{EXPO}(36)$ & 10+WEIB(9.27, 0.295) \\
\hline 15 & $4.5+\mathrm{EXPO}(15.5)$ & $5+\mathrm{EXPO}(23.6)$ & $5+\operatorname{EXPO}(25)$ \\
\hline 16 & 5+WEIB $(10.4,0.459)$ & $20+\operatorname{EXPO}(9.51)$ & $5+\operatorname{WEIB}(3.85,0.241)$ \\
\hline 17 & WEIB $(9.59,0.483)$ & $\operatorname{TRIA}(8,13.7,41)$ & WEIB $(10.5,0.491)$ \\
\hline 18 & 5+WEIB $(13.2,0.353)$ & $\operatorname{TRIA}(10,14,76)$ & $5+\mathrm{WEIB}(0.687,0.256)$ \\
\hline 19 & $\operatorname{UNIF}(5,41)$ & $\operatorname{TRIA}(30,46.5,96)$ & $30+\operatorname{EXPO}(55)$ \\
\hline 20 & 5+WEIB $(10.4,0.459)$ & 20+EXPO(9.51) & 5+WEIB(4.01,0.244) \\
\hline 21 & $\operatorname{EXPO}(27.5)$ & $5+\operatorname{EXPO}(22.5)$ & $56 * \operatorname{BETA}(0.526,0.946)$ \\
\hline 22 & $\operatorname{WEIB}(30.8,0.747)$ & $20+81 * \operatorname{BETA}(0.899,1.26)$ & $\operatorname{TRIA}(19.5,43.5,101)$ \\
\hline 23 & $\operatorname{EXPO}(14.5)$ & $10+\operatorname{EXPO}(52)$ & $\operatorname{TRIA}(5,68,95)$ \\
\hline 24 & $10+85 * \operatorname{BETA}(0.545,0.741)$ & $\operatorname{TRIA}(35,53,71)$ & $10+121 * \operatorname{BETA}(1.44,0.825)$ \\
\hline 25 & $\operatorname{EXPO}(43.4)$ & $25+\operatorname{EXPO}(24.1)$ & $5+\mathrm{WEIB}(40,0.573)$ \\
\hline
\end{tabular}

It is important to mention that some of the activities were represented using a normal distribution. In those cases, in order to eliminate the probability of a negative activity time, a truncated normal distribution was implemented. Since these distributions were fitted using real hospital data, the close to zero values represent that the activity was interrupted due to an unexpected event. For example, if patient's condition worsens during the recovery, the patient must be transferred to the intensive care unit.

The number of hospitalisation days was fitted using empirical distributions. An example of one of the categories is presented in Table II. It shows that a patient of surgery category 8 can be hospitalised 1 day with a probability $0.29,2$ days with a probability 0.28 , etc. One specific table was created for each of the 25 surgeries types. For space limitations, only a single table is presented.

\subsection{Simulation model}

A simulation model using the discrete event simulation software Arena 12.0 from the Rockwell Automation Company was created to represent the surgical patient flow. The patient arrival is basically a queue containing the batch of patients scheduled for surgery. A patient will leave the queue and enter the hospital only if a bed is available. The patient will 
stay at the bed for their turn in the surgical schedule. In that moment if the operating room is available the patient will be assigned to it. After the surgery, if the recovery bed is available, the patient will be transported to it, otherwise he has to wait in the operating room, blocking the next surgery. Finally after recovery, the patient is transported to the hospitalisation bed, where stays for the amount of days that corresponds to that particular patient category.

Table II: Empirical distribution for hospitalisation days for surgery category 8.

\begin{tabular}{|c|c|c|}
\hline Days & Prob. & Cumulative prob. \\
\hline 1 & 0.29 & 0.29 \\
\hline 2 & 0.28 & 0.57 \\
\hline 3 & 0.21 & 0.78 \\
\hline 4 & 0.06 & 0.84 \\
\hline 5 & 0.02 & 0.86 \\
\hline 6 & 0.04 & 0.9 \\
\hline 7 & 0.04 & 0.94 \\
\hline 8 & 0.02 & 0.96 \\
\hline 9 & 0 & 0.96 \\
\hline 10 & 0.04 & 1 \\
\hline
\end{tabular}

The human resources, such as doctors and nurses, were not considered in the model. This assumes that operating room availability implies human resources availability as well. This assumption could be a limitation if the objective of the study is to evaluate the effect that human resources have in the system's performance. In this study these variables are considered fixed since the objective was to evaluate the effect of different patient's schedules.

\subsection{Simulation conditions}

A simulation experiment consists of running a batch of patients until the last patient who is scheduled in that batch leaves the hospital. The measure of performance that was used was the time required to process a batch of patients. A total of 20 replications was required to obtain a 0.95 confidence interval with a $\pm 5 \%$ half-width, which means that each schedule explored during the optimisation process was run 20 times. Two weeks was used as warm-up period to run the experiment in a steady-state process because other patients were using the beds and operating rooms. Each simulation run ended when the last scheduled patient left the hospital.

\subsection{Model validation}

To determine if the simulation model emulated the behaviour of the real system, a set of validation experiments were performed. Ten different months were selected from the hospital data base to calculate the time that was required to complete the surgery process, which includes recovery and hospitalisation. The simulation model was initialised with the same patient schedule and surgery categories. The results show that a significant difference did not exist between the real system and the simulation model.

\section{RESULTS}

The optimisation process requires the definition of the simulated annealing parameters, initial temperature and cooling temperature. Tree alternatives were chosen for each parameter, as shown in Table III. 
Table III: Simulated annealing parameters.

\begin{tabular}{|c|c|}
\hline Initial temperature & Cooling temperature \\
\hline 3000 & 10 \\
\hline 2000 & 5 \\
\hline 1000 & 1 \\
\hline
\end{tabular}

Several experiments using all the combinations of these parameters were performed. The best results were obtained when the initial temperature and cooling temperature were set at 1000 degrees and 1 degree, respectively. The stopping criterion was set as 0 degrees, which means that the algorithm will continue until the final temperature reaches 0 degrees.

The experimentation process consisted of analysing the simulated annealing algorithm performance against three classic dispatching rules: Shortest Processing Time (SPT), Longest Processing Time (LPT) and First-In, First-Out (FIFO). These rules were selected because are very simple to understand and to implement by hospital personnel. Many other more sophisticated dispatching rules or even optimisation algorithms could have been considered in this work; however the orientation of this study was to show how different techniques could have a significant effect in the hospital performance. Three different experiments were performed that considered 85 patients, 170 patients and 265 patients, respectively.

\subsection{Experiment with 85 patients}

This experiment was performed 20 times by considering different random seeds to simulate different sets of patients with different characteristics, where from each run, a patient schedule was obtained. Each patient schedule was replicated 10 times to obtain statistical significance. The results for the Cmax indicator expressed in minutes for each one of the four heuristics are presented in Table IV and in Fig. 5.

Table IV: Cmax results for experiment 1.

\begin{tabular}{|l|r|r|r|r|}
\hline Parameter & \multicolumn{1}{|c|}{ SA } & \multicolumn{1}{c|}{ SPT } & \multicolumn{1}{c|}{ LPT } & \multicolumn{1}{c|}{ FIFO } \\
\hline Mean & 21214 & 23125 & 24750 & 26337 \\
\hline Median & 21431 & 23433 & 24357 & 25993 \\
\hline Standard deviation & 988 & 2114 & 2445 & 2834 \\
\hline Minimum & 19410 & 18787 & 20598 & 21417 \\
\hline Maximum & 22900 & 26655 & 29304 & 31250 \\
\hline
\end{tabular}

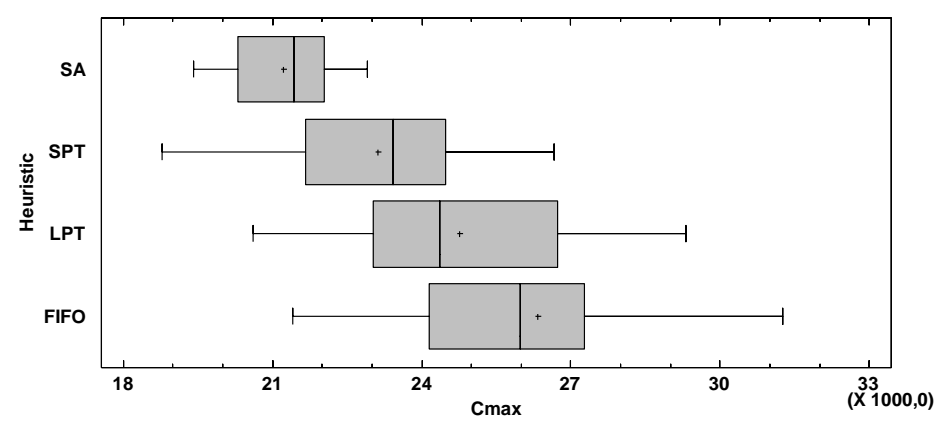

Figure 5: Box plot for experiment 1.

Table IV shows that on average, a total of 21214 minutes is required to process the entire batch of 85 patients using the simulated annealing approach, which is equivalent to 14.7 days. The worst heuristic was the FIFO approach, which required 26337 minutes (18.3 days). It is important to mention that the FIFO approach is equivalent to the scheduling strategy used by 
the hospital. The difference between the simulated annealing and FIFO heuristic is 3.6 days, which corresponds to a $20 \%$ time reduction. Fig. 5 shows the box plots of the four approaches. Based on the mean values, the ranking is SA, SPT, LPT, FIFO. To determine a ranking with significant difference among the four heuristics, it is necessary to perform an allpairwise comparison [23]. This approach compares all the possible pairs and constructs confidence intervals for the mean difference. To obtain an overall confidence of $1-\alpha$, it is necessary to construct each individual confidence interval using the Bonferroni inequality [26]. In this case, with four treatments a total of $k \times(k-1) / 2=6$ pairs are possible, which means that each confidence interval should be constructed with a $1-\alpha / 6$ confidence level to obtain an overall confidence of $1-\alpha$. For this study, an overall confidence of 0.9 was chosen, which implies a 0.983 confidence level for each individual comparison. Table V shows the results of this multiple comparison using the paired- $t$ test.

Table V: All-pairwise comparison confidence intervals for experiment 1.

\begin{tabular}{|l|c|c|c|c|c|c|c|c|c|}
\cline { 2 - 10 } \multicolumn{1}{c|}{} & \multicolumn{3}{c|}{ SPT } & \multicolumn{3}{c|}{ LPT } & \multicolumn{3}{c|}{ FIFO } \\
\cline { 2 - 10 } \multicolumn{1}{c|}{} & LL & Mean & UL & LL & Mean & UL & LL & Mean & \multicolumn{1}{c|}{ UL } \\
\hline SA & -2890 & -1912 & -934 & -4685 & -3537 & -2388 & -6810 & -5124 & -3437 \\
\hline SPT & & & -3063 & -1625 & -187 & -5305 & -3212 & -1119 \\
\hline LPT & & & & & -4010 & -1587 & 836 \\
\hline
\end{tabular}

Table V presents the six confidence intervals constructed with a 0.983 confidence level. The table shows the lower limit, mean and upper limit of the confidence interval for the difference between each pair of heuristics. For example, the comparison between simulated annealing and SPT heuristic resulted in a confidence interval with a mean difference of -1912 minutes with lower and upper limits of -2890 and -934 minutes, respectively. Because the interval does not contain the zero, this result indicates a significant difference between the two heuristics. The negative values imply that the Cmax for the SA approach is less than the Cmax of the STP heuristic. On average, this difference is 1912 minutes. The only confidence interval that contains the zero is the LTP-FIFO comparison, which indicates there is no significant difference between both heuristics. By analysing the complete table, it is possible to conclude with an overall confidence level of 0.9 that the best performance is obtained with the SA approach, followed by the SPT heuristic and finally, in third place, LPT and FIFO with no significant difference.

\subsection{Experiment with 170 patients}

In this experiment, a batch of 170 patients was considered. The same analysis was performed using the SA, SPT, LPT and FIFO heuristics. The results for the Cmax indicator expressed in minutes for each of the four heuristics are presented in Table VI and in Fig. 6.

Table VI: Cmax results for experiment 2.

\begin{tabular}{|l|r|r|r|r|}
\hline Parameter & \multicolumn{1}{|c|}{ SA } & \multicolumn{1}{c|}{ SPT } & \multicolumn{1}{c|}{ LPT } & \multicolumn{1}{c|}{ FIFO } \\
\hline Mean & 44259 & 48528 & 50062 & 53396 \\
\hline Median & 45100 & 47080 & 49182 & 53459 \\
\hline Standard deviation & 4526 & 5714 & 7046 & 6973 \\
\hline Minimum & 34264 & 37908 & 38505 & 43146 \\
\hline Maximum & 51807 & 60915 & 64269 & 68598 \\
\hline
\end{tabular}




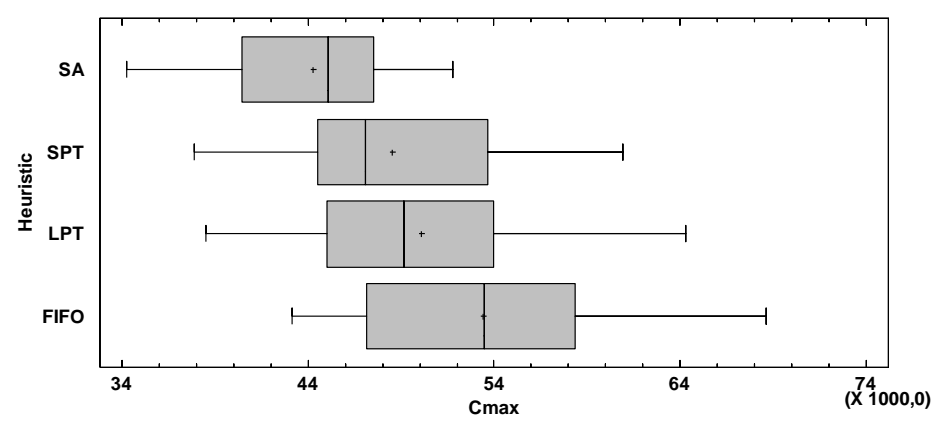

Figure 6: Box plot for experiment 2.

Table VI shows that on average, a total of 44259 minutes are required to process the entire batch of 170 patients using the simulated annealing approach, which is equivalent to 30.7 days. The worst heuristic was the FIFO approach, which required 53396 minutes (37 days). The difference between the simulated annealing and FIFO heuristics is 6.7 days, which corresponds to a $17 \%$ of time reduction. Fig. 6 presents the box plots of the four approaches, which shows the same behaviour as experiment 1 , though the box plots do overlap more. The same procedure of multiple comparisons was applied to this experiment. The results are presented in Table VII.

Table VII: All pairwise comparison confidence intervals for experiment 2.

\begin{tabular}{|l|c|c|c|c|c|c|c|c|c|}
\cline { 2 - 10 } \multicolumn{1}{c|}{} & \multicolumn{3}{c|}{ SPT } & \multicolumn{3}{c|}{ LPT } & \multicolumn{3}{c|}{ FIFO } \\
\cline { 2 - 10 } \multicolumn{1}{c|}{} & LL & Mean & UL & LL & Mean & UL & LL & Mean & \multicolumn{1}{c|}{ UL } \\
\hline SA & -6441 & -4269 & -2097 & -8902 & -5803 & -2704 & -12868 & -9136 & -5404 \\
\hline SPT & & & -5246 & -1534 & 2179 & -7906 & -4867 & -1829 \\
\hline LPT & & & & & -8787 & -3334 & 2120 \\
\hline
\end{tabular}

By analysing the complete table, it is possible to conclude with an overall confidence level of 0.9 that the best performance was obtained with the SA approach. This result is the same as that obtained in experiment 1; however, it is not possible to determine the second position in the ranking because the intervals include the zero in the SPT-LPT and LPT-FIFO intervals.

\subsection{Experiment with 255 patients}

The results for the Cmax indicator expressed in minutes for each of the four heuristics are presented in Table VIII and in Fig. 7.

Table VIII: Cmax results for experiment 3.

\begin{tabular}{|l|r|r|r|r|}
\hline Parameter & \multicolumn{1}{|c|}{ SA } & \multicolumn{1}{c|}{ SPT } & \multicolumn{1}{c|}{ LPT } & \multicolumn{1}{c|}{ FIFO } \\
\hline Mean & 62460 & 64933 & 69606 & 75190 \\
\hline Median & 62347 & 63745 & 69343 & 74640 \\
\hline Standard deviation & 2083 & 4700 & 6613 & 7073 \\
\hline Minimum & 58951 & 57206 & 57413 & 62006 \\
\hline Maximum & 66542 & 75241 & 81467 & 89478 \\
\hline
\end{tabular}

Table VIII shows that on average, a total of 62460 minutes was required to process the entire batch of 255 patients using the simulated annealing approach, which is equivalent to 43.4 days. The worst heuristic was the FIFO approach, which required 75190 minutes $(52.2$ days). The difference between the simulated annealing and FIFO heuristics is 8.8 days, which 
corresponds to a $17 \%$ time reduction. Fig. 7 presents the box plots of the four approaches, which show the same behaviour from experiments 1 and 2 . The multiple comparisons results are presented in Table IX.

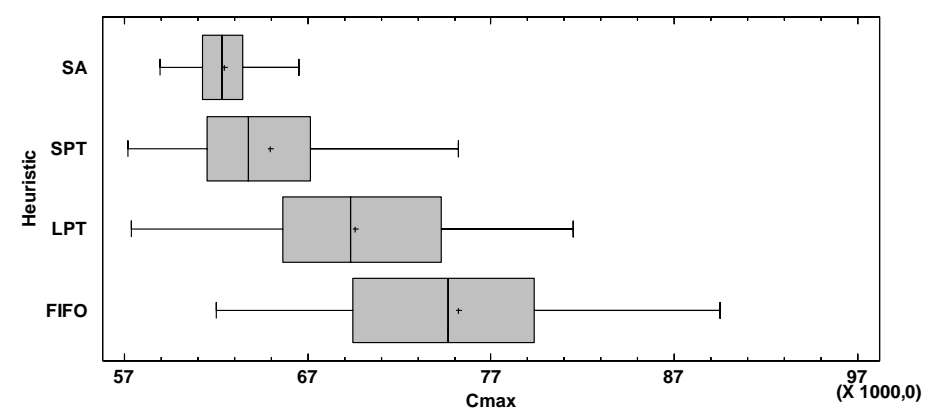

Figure 7: Box plot for experiment 3.

Table IX: All pairwise comparison confidence intervals for experiment 3.

\begin{tabular}{|l|c|c|c|r|c|c|c|c|c|}
\cline { 2 - 10 } \multicolumn{1}{c|}{} & \multicolumn{3}{c|}{ SPT } & \multicolumn{3}{c|}{ LPT } & \multicolumn{3}{c|}{ FIFO } \\
\cline { 2 - 10 } \multicolumn{1}{c|}{} & LL & Mean & UL & LL & Mean & UL & LL & Mean & \multicolumn{1}{c|}{ UL } \\
\hline SA & -4700 & -2473 & -246 & -10636 & -7146 & -3656 & -16489 & -12730 & -8971 \\
\hline SPT & & & & -8970 & -4673 & -375 & -14380 & -10257 & -6133 \\
\hline LPT & \multicolumn{4}{|c}{} & & & -10338 & -5584 & -831 \\
\hline
\end{tabular}

Table IX shows that all the confidence intervals are significant. Thus, it is possible to conclude with a 0.9 overall confidence level that the best performance was obtained by the SA heuristic, the second best is the SPT approach, the third best is LPT, and last place is the FIFO rule.

\section{CONCLUSIONS}

This paper presents a methodology based on a simulated annealing and discrete-event simulation that can determine good schedules for surgery patients. The results showed that it is possible to reduce the time required to process a batch of patients by $18 \%$ when the SA search method is used instead of the FIFO approach (the current hospital method). This is considering the average of the 3 experiments. The improvement was independent of batch size because essentially, the same conclusions and improvements were obtained in the 3 experiments. The second best method was the SPT rule with a $12 \%$ improvement compared with that of the FIFO rule. The LPT rule performed similarly to the FIFO approach in two of the three experiments, which shows that it is not a good rule for this particular problem. The most important conclusion of this paper is that it is possible to solve the stochastic operating room scheduling problem from a practical point of view that uses search algorithms and simulation. The problem representation was realistic, which included the primary steps of a surgical process, particularly variables such as bed availability, a resource that frequently is the bottleneck of the process. It is important to mention that many other algorithms could be used, such as genetic algorithms or evolutionary strategies; however, these population-based metaheuristics can require a larger amount of solutions to perform a good search compared with local search techniques, such the SA approach used in this work. This issue is extremely important to consider because each schedule that was evaluated during the search corresponds to a simulated scenario that must be replicated $n$ times, which consumes a significant amount of time and makes it difficult to explore extensive search spaces. It is noteworthy that generally healthcare personnel performing these tasks are unaware of the potential operational 
effect that the application of different patient schedules may have. They mainly consider clinical factors when preparing a schedule. This study clearly shows the effect that can be generated on an operational indicator when an optimisation heuristic as simulated annealing or other simple dispatching rules are used.

Future work will focus on using other local search algorithms, such as the tabu search, to evaluate its performance. Although the case study presented here was for a small hospital, the methodology does not significantly differ when larger hospitals are analysed because the patient process is essentially the same. One important difference that could be encountered in a larger institution is related to the human resources availability. This issue incorporates an additional dimension to the problem that must be added to the simulation model logic.

\section{REFERENCES}

[1] Cardoen, B.; Demeulemeester, E.; Beliën, J. (2010). Operating room planning and scheduling: A literature review, European Journal of Operational Research, Vol. 201, No. 3, 921-932, doi:10.1016/j.ejor.2009.04.011

[2] Blake, J. T.; Carter, M. W. (1997). Surgical process scheduling: a structured review, Journal of the Society for Health Systems, Vol. 5, No. 3, 17-30

[3] Kaban, A. K.; Othman, Z.; Rohmah, D. S. (2012). Comparison of dispatching rules in job-shop scheduling problem using simulation: A case study, International Journal of Simulation Modelling, Vol. 11, No. 3, 129-140, doi:10.2507/IJSIMM11(3)2.201

[4] Korytkowski, P.; Wisniewski, P.; Rymaszewski, S. (2013). An evolutionary simulation-based optimization approach for dispatching scheduling, Simulation Modelling Practice and Theory, Vol. 35, 69-85, doi:10.1016/j.simpat.2013.03.006

[5] Geyik, F.; Dosdogru, A. T. (2013). Process plan and part routing optimization in a dynamic flexible job shop scheduling environment: an optimization via simulation approach, Neural Computing and Applications, Vol. 23, No. 6, 1631-1641, doi:10.1007/s00521-012-1119-7

[6] Banditori, C.; Cappanera, P.; Visintin, F. (2013). A combined optimization-simulation approach to the master surgical scheduling problem, IMA Journal of Management Mathematics, Vol. 24, No. 2, 155-187, doi:10.1093/imaman/dps033

[7] Chow, V. S.; Puterman, M. L.; Salehirad, N.; Huang, W.; Atkins, D. (2011). Reducing surgical ward congestion through improved surgical scheduling and uncapacitated simulation, Production and Operations Management, Vol. 20, No. 3, 418-430, doi:10.1111/j.1937-5956.2011.01226.x

[8] Helm, J. E.; Lapp, M.; See, B. D. (2010). Characterizing and effective hospital admission scheduling and control management system: A genetic algorithm approach, Proceedings of the 2010 Winter Simulation Conference, 2387-2398, doi:10.1109/WSC.2010.5678935

[9] Sun, Y.; Li, X. (2011). Optimizing surgery start times for a single operating room via simulation, Proceedings of the 2011 Winter Simulation Conference, 1306-1313, doi:10.1109/WSC. $\underline{2011.6147851}$

[10] Koeleman, P.; Koole, G. (2012). Appointment scheduling using optimisation via simulation, Proceedings of the 2012 Winter Simulation Conference, 251-255, doi:10.1109/WSC.2012. $\underline{6465186}$

[11] Harper, P. R.; Gamlin, H. M. (2003). Reduced outpatient waiting times with improved appointment scheduling: a simulation modelling approach, OR Spectrum, Vol. 25, No. 2, 207222, doi:10.1007/s00291-003-0122-x

[12] Dexter, F.; Macario, A.; Traub, R. D.; Hopwood, M.; Lubarsky, D. A. (1999). An operating room scheduling strategy to maximize the use of operating room block time: computer simulation of patient scheduling and survey of patients' preferences for surgical waiting time, Anesthesia \& Analgesia, Vol. 89, No. 1, 7-20, doi:10.1213/00000539-199907000-00003

[13] Lee, S.; Yih, Y. (2010). Analysis of an open access scheduling system in outpatient clinics: A simulation study, Simulation, Vol. 86, No. 8-9, 503-518, doi:10.1177/0037549709358295

[14] Arnaout, J.-P. (2010). Heuristics for the maximization of operating rooms utilization using simulation, Simulation, Vol. 86, No. 8-9, 573-583, doi:10.1177/0037549709352497 
[15] Sciomachen, A.; Tanfani, E.; Testi, A. (2005). Simulation models for optimal schedules of operating theatres, International Journal of Simulation, Vol. 6, No. 12-13, 26-34

[16] Metropolis, N.; Rosenbluth, A. W.; Rosenbluth, M. N.; Teller, A. H.; Teller, E. (1953). Equation of state calculations by fast computing machines, The Journal of Chemical Physics, Vol. 21, No 6, 1087-1092

[17] Kirkpatrick, S.; Gelatt, C. D.; Vecchi, M. P. (1983). Optimization by simulated annealing, Science, Vol. 220, No. 4598, 671-680

[18] Zhang, R.; Wu, C. (2011). A simulated annealing algorithm based on block properties for the job shop scheduling problem with total weighted tardiness objective, Computers \& Operations Research, Vol. 38, No. 5, 854-867, doi:10.1016/j.cor.2010.09.014

[19] Chen, P.-H.; Shahandashti, S. M. (2009). Hybrid of genetic algorithm and simulated annealing for multiple project scheduling with multiple resource constraints, Automation in Construction, Vol. 18, No. 4, 434-443, doi:10.1016/j.autcon.2008.10.007

[20] Aksoy, A.; Ozturk, N. (2010). Simulated annealing approach in scheduling of virtual cellular manufacturing in the automotive industry, International Journal of Vehicle Design, Vol. 52, No. 1-4, 82-95, doi:10.1504/IJVD.2010.029637

[21] Kaplan, S.; Rabadi, G. (2013). Simulated annealing and metaheuristic for randomized priority search algorithms for the aerial refuelling parallel machine scheduling problem with due date-todeadline windows and release times, Engineering Optimization, Vol. 45, No. 1, 67-87, doi:10.1080/0305215X.2012.658783

[22] Mirsanei, H. S.; Zandieh, M.; Moayed, M. J.; Khabbazi, M. R. (2011). A simulated annealing algorithm approach to hybrid flow shop scheduling with sequence-dependent setup times, Journal of Intelligent Manufacturing, Vol. 22, No. 6, 965-978, doi:10.1007/s10845-009-0373-8

[23] Mattila, V.; Virtanen, K. (2014). Maintenance scheduling of a fleet of fighter aircraft through multi-objective simulation-optimization, Simulation, Vol. 90, No. 9, 1023-1040, doi:10.1177/ $\underline{0037549714540008}$

[24] Zhang, Y.; Brown, D. (2014). Simulation optimization of police patrol districting plans using response surfaces, Simulation, Vol. 90, No. 6, 687-705, doi:10.1177/0037549714533159

[25] Haridass, K.; Valenzuela, J.; Yucekaya, A. D.; McDonald, T. (2014). Scheduling a log transport system using simulated annealing, Information Sciences, Vol. 264, 302-316, doi:10.1016/ j.ins.2013.12.005

[26] Law, A. M. (2007). Simulation Modeling and Analysis, McGraw-Hill, Boston 